\title{
Short communication: Improving the activity of bile salt hydrolases in Lactobacillus casei based on in silico molecular docking and heterologous expression
}

\author{
Zhi-Qiang Xiong, ${ }^{*}$ Qiao-Hui Wang, ${ }^{*}$ Ling-Hui Kong, ${ }^{*}$ Xin Song, ${ }^{*}$ Guang-Qiang Wang, ${ }^{*}$ Yong-Jun Xia, \\ Hui Zhang, ${ }^{*}$ Yong Sun, $†$ and Lian-Zhong $\mathrm{Ai}^{* 1}$ \\ *Shanghai Engineering Research Center of Food Microbiology, School of Medical Instruments and Food Engineering, \\ University of Shanghai for Science and Technology, Shanghai 200093, China \\ †Yangzhou Sanhesimei Pickles Co. Ltd., Yangzhou 225000, China
}

\begin{abstract}
Bile salt hydrolase (BSH) plays an essential role in the cholesterol-removing effect of lactic acid bacteria, which hydrolyze conjugated bile salts to amino acid and deconjugated bile salts. However, Lactobacillus casei lacks the bsh gene, which may make it highly sensitive to bile salt stress. We wanted to improve the BSH activity of $L$. casei for various food-industry applications (e.g., milk fermentation). Plate assay testing indicated that Lactobacillus plantarum AR113 has the highest BSH activity. We cloned and sequenced 4 bsh genes from the genome of $L$. plantarum AR113. Structure modeling and molecular docking of BSH indicated that BSH1 and BSH3 could react efficiently with bile salts, so we selected BSH1 and BSH3 for heterologous expression in L. casei. Compared with single expression of BSH1 or BSH3, co-expression of both protein sequences showed the highest hydrolysis activity by HPLC analysis. Our results suggested that heterologous expression of BSH in L. casei can significantly improve host activity against bile salts, and in silico molecular docking could be an efficient method of rapid screening for BSH with high activity.
\end{abstract}

Key words: bile salt hydrolase, Lactobacillus casei, molecular docking, heterologous expression

\section{Short Communication}

Lactobacillus casei has been widely applied in the food industry as an acid-producing starter culture for milk fermentation and as an adjunct culture to intensify and accelerate flavor development in ripening cheese. It is also a probiotic organism with public health benefits, such as decreasing incidence of gastrointestinal diseases

Received July 27, 2016.

Accepted September 2, 2016.

${ }^{1}$ Corresponding author: ailianzhong@hotmail.com caused by pathogenic bacteria, modulating the immune system, and reducing the risk of bladder cancer. However, L. casei faces a number of stresses from industrial processes and during gastrointestinal transit, including bile salts stress.

In nature, probiotics have evolved bile salt hydrolase (BSH, EC 3.5.1.2) to deal with bile salt stress. An important enzyme for the removal of cholesterol, BSH catalyzes the release of free amino acids from both conjugated and deconjugated bile salts. However, L. casei lacks the $b s h$ gene and may be highly sensitive to bile salt stress. To improve the ability of $L$. casei to degrade bile salts, we cloned bsh genes from L. plantarum and introduced into L. casei.

The lactic acid bacteria used in this study (Supplementary Table S1; https://doi.org/10.3168/jds.201611720 ) were cultured under anaerobic conditions at $37^{\circ} \mathrm{C}$ in de Man, Rogosa, Sharpe (MRS) medium using an anaerobic system (Whitley DG250 workstation; Don Whitley Scientific Limited, Shipley, UK) with a continuous flow of nitrogen gas. The qualitative BSH activity of lactic acid bacteria was determined by plate assay (Dashkevicz and Feighner, 1989; Jayashree et al., 2014). We grew 10 lactic acid bacteria strains (LC2W, AR302, AR342, JPP2, WCFS1, AR113, AR171, AR300, AR184, and AR328) on MRS agar plates supplemented with $0.37 \mathrm{~g} / \mathrm{L} \mathrm{CaCl}_{2}$ and $5 \mathrm{mM}$ bile salts containing glycocholate (Gc), glycodeoxycholate (Gdc), and glycochenodeoxycholate (Gcdc). The plates were incubated at $37^{\circ} \mathrm{C}$ for $48 \mathrm{~h}$. Bile acid precipitates around the colonies (opaque halo) or the formation of opaque granular white colonies with a silvery shine were considered to be BSH activity (Jayashree et al., 2014). Strains LC2W, AR302, and AR342, which belonged to L. casei, could not grow on the MRS medium supplemented with bile salts, indicating that $L$. casei has low or no BSH activity. Compared with the control (no bile salts in the medium; Figure 1A), strain AR113, which belonged to Lactobacillus plantarum, showed higher BSH activity 

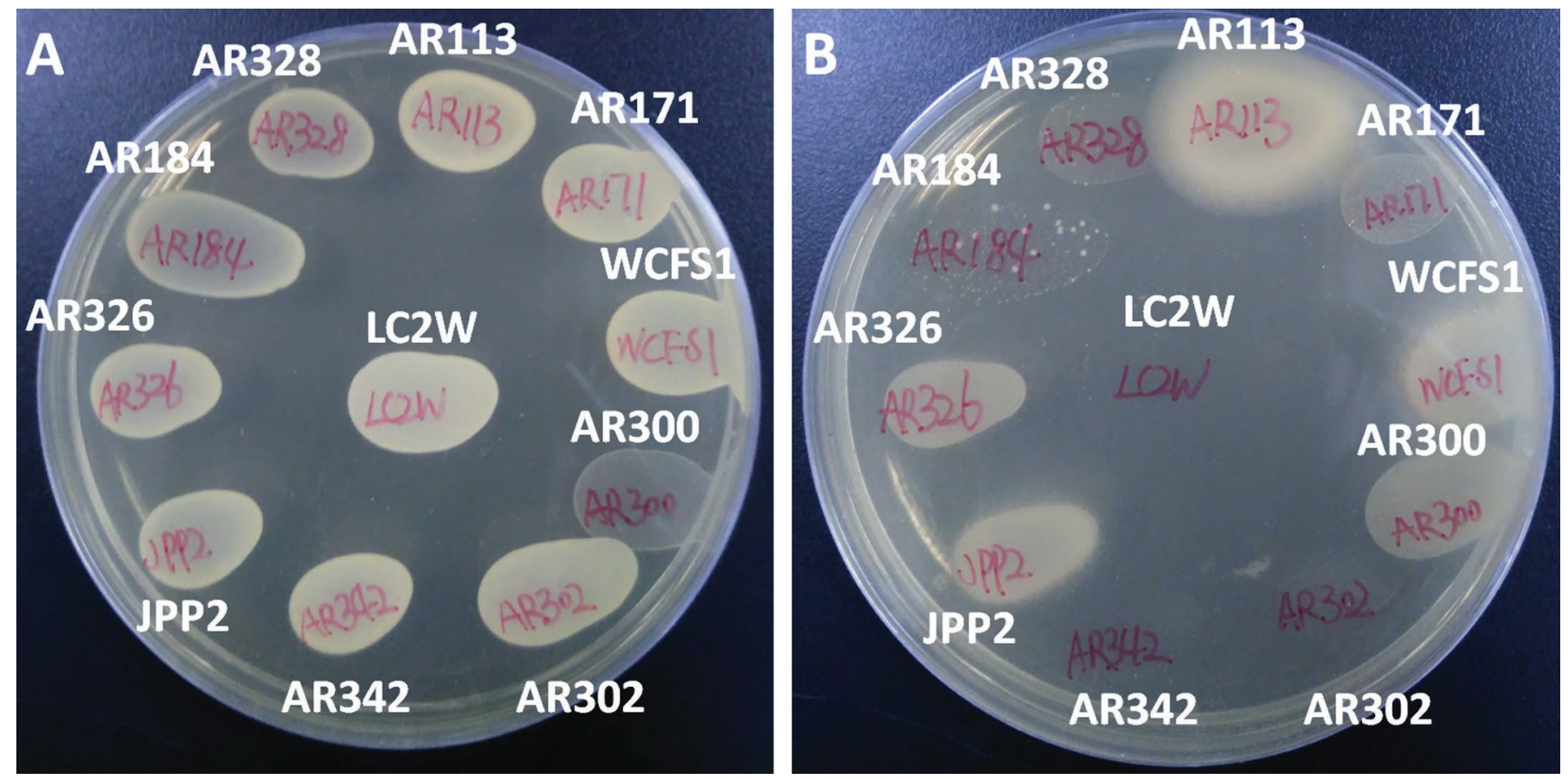

Figure 1. The detection of bile salt hydrolase (BSH) activity using a plate assay. Ten lactic acid bacteria strains (LC2W, AR302, AR342, JPP2, WCFS1, AR113, AR171, AR300, AR184, and AR328) were grown on de Man, Rogosa, and Sharpe (MRS) agar plates with 0.37 g/L CaCl (A) or $0.37 \mathrm{~g} / \mathrm{L} \mathrm{CaCl}{ }_{2}$ and $5 \mathrm{mM}$ bile salt (B) at $37^{\circ} \mathrm{C}$ using an anaerobic system with a continuous flow of nitrogen gas. Bile acid precipitates around the colonies (opaque halo) or the formation of opaque granular white colonies with silvery shine were considered to indicate BSH activity. Color version available online.

based on the size of the opaque halo around the colony (Figure 1B). For this reason, we selected strain AR113 for cloning of bsh genes.

According to a genomic analysis, L. plantarum has 4 bsh genes with different DNA sequences ( $\mathrm{Gu}$ et al., 2014). The 4 bsh genes (designated bsh1, bsh2, bsh3, and bsh4) were amplified via PCR from the DNA of L. plantarum AR113, using primers BSH1-F/BSH1$\mathrm{R}$, BSH2-F/BSH2-R, BSH3-F/BSH3-R, and BSH4F/BSH4-R (Supplementary Table S2; https://doi. org/10.3168/jds.2016-11720), respectively. The primers were designed based on the published DNA sequence of bsh genes from L. plantarum WCFS1 (NC_004567.2). We inserted the PCR products (Supplementary Figure S1; https://doi.org/10.3168/jds.2016-11720) into lactic acid bacteria shuttle vector pMG36e, containing a constitutive promoter $\mathrm{P}_{32}$ (Biswas et al., 2008) and using the ClonExpress 1-step cloning kit (Vazyme Biotech, Nanjing, China) to construct plasmids pWQH01 to pWQH04. Plasmids were transformed into Escherichia coli TOP10 and then sequenced by Sangon Biotech (Shanghai, China). The results of sequencing showed that bsh1-4 contained single open reading frames of $975,1,017,987$, and 954 nucleotides, respectively (Supplementary Text S1; https://doi.org/10.3168/ jds.2016-11720). We found that bsh1-4, all encoding for
BSH, were similar to bsh1-4 from L. plantarum WCFS1 and L. plantarum JPP2, with high identities (98-99\%) using National Center for Biotechnology Information (NCBI) Blastx analysis (http://blast.ncbi.nlm.nih. gov/Blast.cgi). The deduced proteins BSH1-4 (Supplementary Text S2; https://doi.org/10.3168/jds.201611720) had theoretical molecular weights of 37.06 , $37.54,36.17$, and $35.64 \mathrm{kDa}$ and isoelectric points of 5.13, 5.93, 5.20, and 8.30, respectively, using ExPASy analysis (http://web.expasy.org/compute_pi).

We used the protein sequences of BSH1-4 to construct a phylogenetic tree using a neighbor-joining algorithm with bootstrap replication of 1,000 in MEGA6 software (Tamura et al., 2013). Phylogenetic tree analysis also showed that the BSH of L. plantarum AR113 had the highest sequence identities with the BSH of 27I,N0RuTSDu L. plantarum WCFS1 and L. plantarum JPP2 (Figure 2A). We found that BSH1-4 showed low sequence identities with each other, and had longer genetic distances to each other, but were highly conserved in evolution. We found similar results for the BSH from L. plantarum CGMCC 8198 (Gu et al., 2014). Furthermore, BSH2 and BSH3 were more homologous among strains than BSH1 and BSH4, implying that evolutionary BSH1 and BSH4 may appear earlier than BSH2 and BSH3 (Figure 2A). We performed domain analysis 
of BSH using the NCBI conserved domain search tool (http://www.ncbi.nlm.nih.gov/Structure/cdd/wrpsb. cgi?) and found that BSH1-4 possessed the domains of the Ntn_hydrolase superfamily (accession no. cl00467), Ntn_penicillin V acylase (accession no. cd00542), YxeI (accession no. COG3049), and CBAH (accession no. pfam02275), suggesting that they have the potential to hydrolyze conjugated bile salts to amino acids and deconjugated bile salts. Only BSH3 had a histidinol dehydrogenase domain (accession no. PRK12447).

We predicted the in silico model structures of BSH using the Swiss-Model server (Arnold et al., 2006; Bordoli et al., 2009). The structures of BSH1-4 were predicted to have 17.0, 14.7, 17.1, and $13.9 \%$ o-helix and 30.2, 30.4, 28.4, and 27.5\% $\beta$-sheet, respectively (Figure 2B). Transmembrane segment prediction by Phobius server (http://phobius.sbc.su.se) indicated that there was no transmembrane region in BSH (Supplementary Figure S2; https://doi.org/10.3168/jds.2016-11720), suggesting that BSH are soluble proteins. We validated the predicted structures using a protein structure validation tool (http://psvs-1_5-dev.nesg.org). The results suggested that the structure models of BSH could be used for molecular docking simulation (Supplementary Figure S3; https://doi.org/10.3168/jds.2016-11720). We simulated in silico molecular docking between BSH1-4 and bile salts using a docking server (Bikadi and Hazai, 2009). We downloaded 3-dimensional models of ligands Gc, Gdc, and Gcdc directly from the NCBI PubChem Compound database (http://www.ncbi.nlm. nih.gov/pccompound) and carried out all docking runs using the default parameters of the docking server.

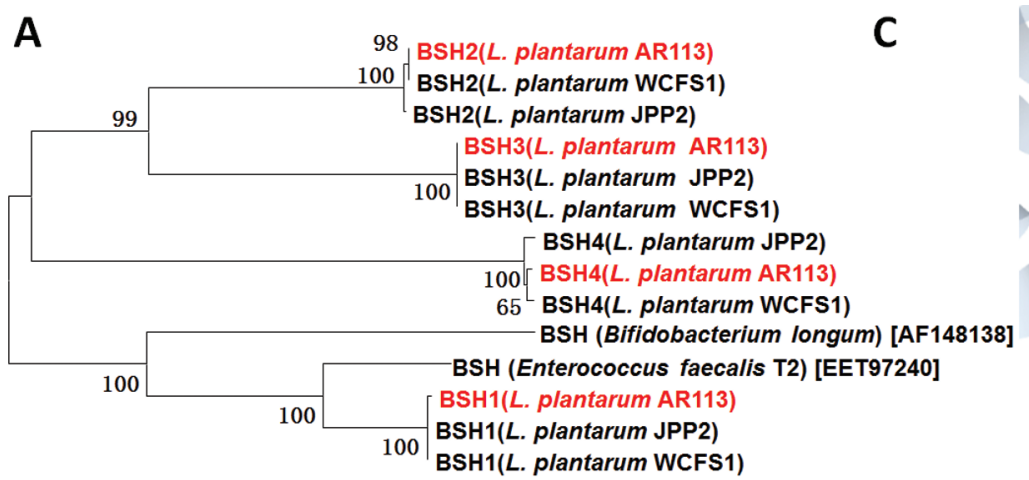

B $\quad$ BSH1

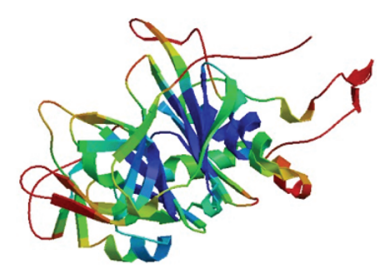

BSH3

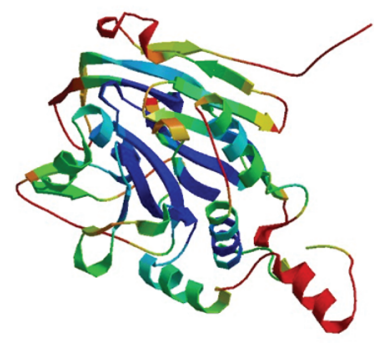

D

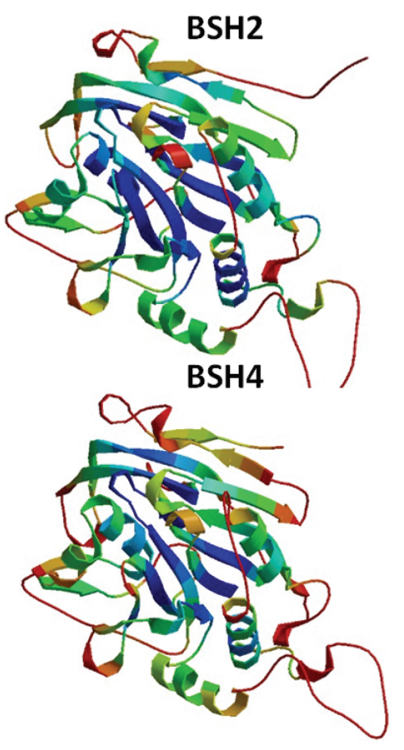

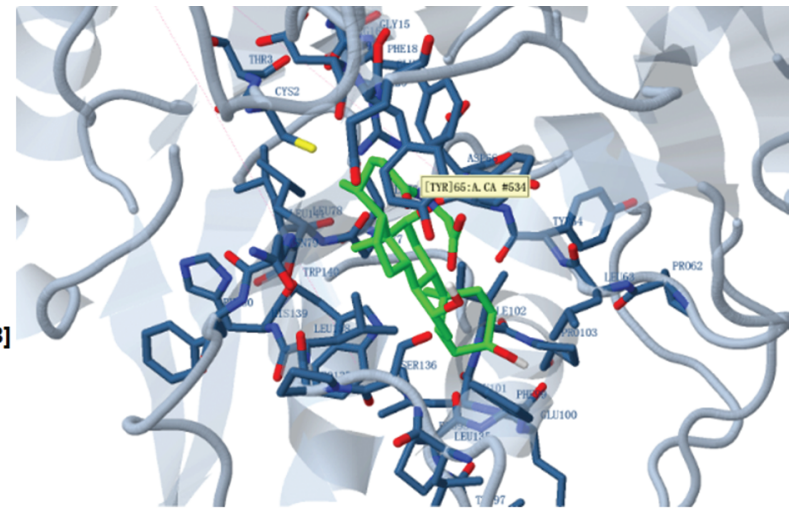

- Glycochenodeoxycholate (Gcdc)

Glycocholate (Gc)

Glycodeoxycholate (Gdc)

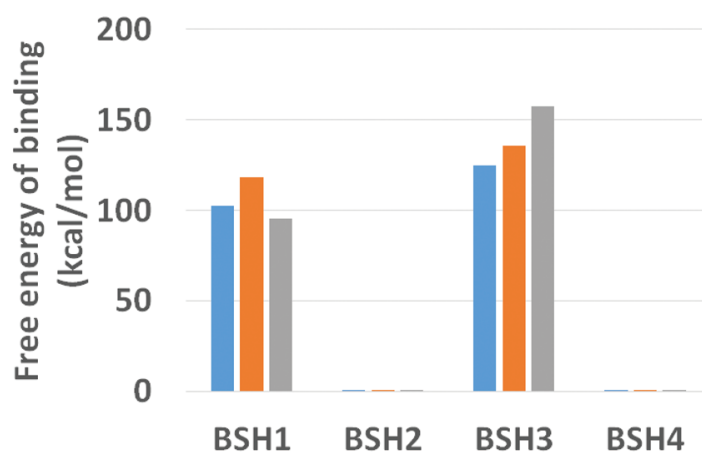

Figure 2. Bioinformatics analysis of bile salt hydrolase (BSH) from Lactobacillus plantarum AR113. (A) Phylogenetic analysis of BSH1-4. The protein sequences were used to construct a phylogenetic tree using a neighbor-joining algorithm with bootstrap replication of 1,000 and MEGA6 software (Tamura et al., 2013). (B) In silico tertiary structure modeling results for BSH1-4 using the Swiss-Model server (Arnold et al., 2006; Bordoli et al., 2009). (C) In silico molecular docking of glycocholate to BSH1 simulated by a docking server (Bikadi and Hazai, 2009). (D) Estimated binding free energy (kcal/mol) of the molecular docking of BSH1-4 and bile salts (glycocholate, Gc; glycodeoxycholate, Gdc; and glycochenodeoxycholate, Gcdc) by the docking server. Color version available online. 
The results indicated that BSH1 and BHS3 had higher binding free energy than BSH2 and BSH4 (Figure 2D and Supplementary Table S3; https://doi.org/10.3168/ jds.2016-11720). The binding free energy of BSH1 with Gcdc, Gc, and Gdc was 102.36, 118.36, and $95.58 \mathrm{kcal} /$ $\mathrm{mol}$, and of BSH2 was 0.91, 0.17, and $0.61 \mathrm{kcal} / \mathrm{mol}$, respectively (Figure 2D). Therefore, BSH1 and BHS3 could bind more efficiently with ligands than BSH2 and BSH4 (Figure 2C and 2D; Supplementary Table S3). Given the similar docking results of BSH1 and BSH3, they were chosen for heterologous expression in L. casei.

The $L$. casei LC2W strain was recently isolated from traditional dairy products in Inner Mongolia, China, and it has been proven to be a multifunctional probiotic that can produce antihypertensive exopolysaccharides (Ai et al., 2008; Xu et al., 2015; Ai et al., 2016). This strain has been applied in the industrial production of certain dairy products (Chen et al., 2011). The entire genome sequence has been reported for $L$. casei $\mathrm{LC} 2 \mathrm{~W}$ (Chen et al., 2011) and shown not to have bsh genes. To improve the bile-salt degradation ability of L. casei, pWQH01 and pWQH03 containing bsh 1 and bsh 3 genes, respectively, were introduced into $L$. casei LC2W using the electroporation method of Aukrust et al. (1995). The L. casei harboring pWQH01 or pWQH03 were grown individually in MRS broth supplemented with erythromycin $(20 \mu \mathrm{g} / \mathrm{mL})$ and incubated at $37^{\circ} \mathrm{C}$ under static conditions. When the optical density at $600 \mathrm{~nm}$ reached 1.0, cells were harvested by centrifugation at $10,625 \times g$ for $5 \mathrm{~min}$. The cell pellet was resuspended in the buffer $(50 \mathrm{mM} \mathrm{NaAc}, \mathrm{pH} 5.4)$ and sonicated for $20 \mathrm{~min}$. The cell lysate was centrifuged at 12,000 $\mathrm{rpm}$ for $30 \mathrm{~min}$ at $4^{\circ} \mathrm{C}$ and analyzed by SDS-PAGE with Coomassie brilliant blue staining (R250). Compared with $L$. casei harboring empty vector pMG36e (control), those harboring pWQH01 or pWQH03 had a desired band at 36 to $38 \mathrm{kDa}$, suggesting that BSH1 and BSH3 could be heterologously expressed in L. casei at the desired molecular weight (Figure 3A). However, although BSH1 and BSH3 were successfully expressed in $L$. casei, their expression was low. One reason for the low expression may have been that $L$. casei is not a good host for protein expression. Another reason may have been that the constitutive promoter $\mathrm{P}_{32}$ was not suitable for BSH expression.

We mixed $1 \mathrm{~mL}$ of cell lysate with $1 \mathrm{~mL}$ of bile salts (5 mM Gc, Gdc, and Gcdc). The reaction mixtures were incubated at $37^{\circ} \mathrm{C}$ for $6 \mathrm{~h}$. Then, $15 \%$ (wt/vol) trichloroacetic acid was added to stop the reaction, and the mixtures were centrifuged at $10,625 \times g$ for 10 min to obtain the samples. The concentrations of Gc, Gdc, and Gcdc were detected using HPLC with a $\mathrm{C}_{18}$ column (Sepax Bio, Newark, DE; $4.6 \times 150 \mathrm{~mm}$ ) and acetonitrile $/ \mathrm{H}_{2} \mathrm{O}$ binary solvent-delivery gradient elution (0-8 $\mathrm{min}, 25-35 \%$ acetonitrile; 8-30 $\mathrm{min}, 25-48 \%$ acetonitrile; $30-31 \mathrm{~min}, 48-25 \%$ acetonitrile; $31-40$ min, $25 \%$ acetonitrile). One unit of BSH activity (U/ cells per hour) was defined as the cell lysate of $10^{9}$ cells that degraded $1 \mathrm{nmol}$ of bile salt per hour. Compared with the control (no BSH activity), the BSH activity of L. casei/pWQH01 on Gc, Gdc, and Gcdc by overexpression of bsh1 was 48.7, 72.37, and $46.35 \mathrm{U} / \mathrm{mL}$, respectively. The BSH activity of $L$. casei/pWQH03 on Gc, Gdc, and Gcdc by overexpression of bsh3 was 57.7, 2.82 , and $61.5 \mathrm{U} /$ cells per hour, respectively.

To further improve BSH activity in L. casei, we constructed a co-expression plasmid pWQH05 (Supplementary Figure S4; https://doi.org/10.3168/jds.201611720) using the ClonExpress one-step cloning kit (Vazyme Biotech), which contains 2 constitutive promoters, $\mathrm{P}_{23}$ (cloning from plasmid pIB184; Biswas et al., 2008) using primers $\mathrm{p} 23-\mathrm{F} / \mathrm{p} 23-\mathrm{R}$ ) and $\mathrm{P}_{\text {ldh }}$ (the promoter of the lactate dehydrogenase gene, cloning from the genome of $L$. casei using primers pldh-F/pldh-R). The bsh 1 and bsh 3 genes were cloned from the genome of L. plantarum AR113 using primers BSH1-F1/BSH1-R1 and BSH3-F3/BSH3-R3 (Supplementary Table S2), double-digested by restriction endonucleases BamHI/ $E c o$ RI and EcoRV/NaeI, and inserted into pWQH05 to form pWQH06 (Supplementary Figure S4) for the co-expression of BSH1 and BSH3. Then, pWQH06 was transformed into L. casei and BSH expression and activity was detected. The SDS-PAGE results showed that BSH expression was further enhanced by coexpression of BSH1 and BSH3 (Figure 3A). To rapidly evaluate BSH expression levels, we quantified the relative content of $\mathrm{BSH}$ (the relative ratio of $\mathrm{BSH}$ to the standard protein) using the volume analysis method with Quantity One software (BioRad Laboratories, Hercules, CA). The BSH concentrations by single expression of BSH1 or BSH3 were 50.04 and $62.44 \mathrm{mg} / \mathrm{L}$, respectively, and BSH concentration by co-expression of BSH1 and BSH3 reached $87.5 \mathrm{mg} / \mathrm{L}$. Modulation of BSH1 and BSH3 in L. casei significantly improved BSH activity on Gc and Gdc (96.4 and $131.0 \mathrm{U} /$ cells per hour) compared with single expression of $\mathrm{BSH}$, but with no obvious change of BSH activity on Gcdc (Figure $3 \mathrm{~B}$ ). This result suggested that co-expression could further increase BSH activity in L. casei.

Bile salts are synthesized from cholesterol, conjugated with glycine or taurine in the liver, and secreted into the small intestine (Kim and Lee, 2005). The gastrointestinal microbiota have evolved BSH activity to degrade bile salts, playing an essential role in bile detoxification and reducing serum cholesterol levels (Kim and Lee, 2005; Jeun et al., 2010; Patel et al., 2010). 


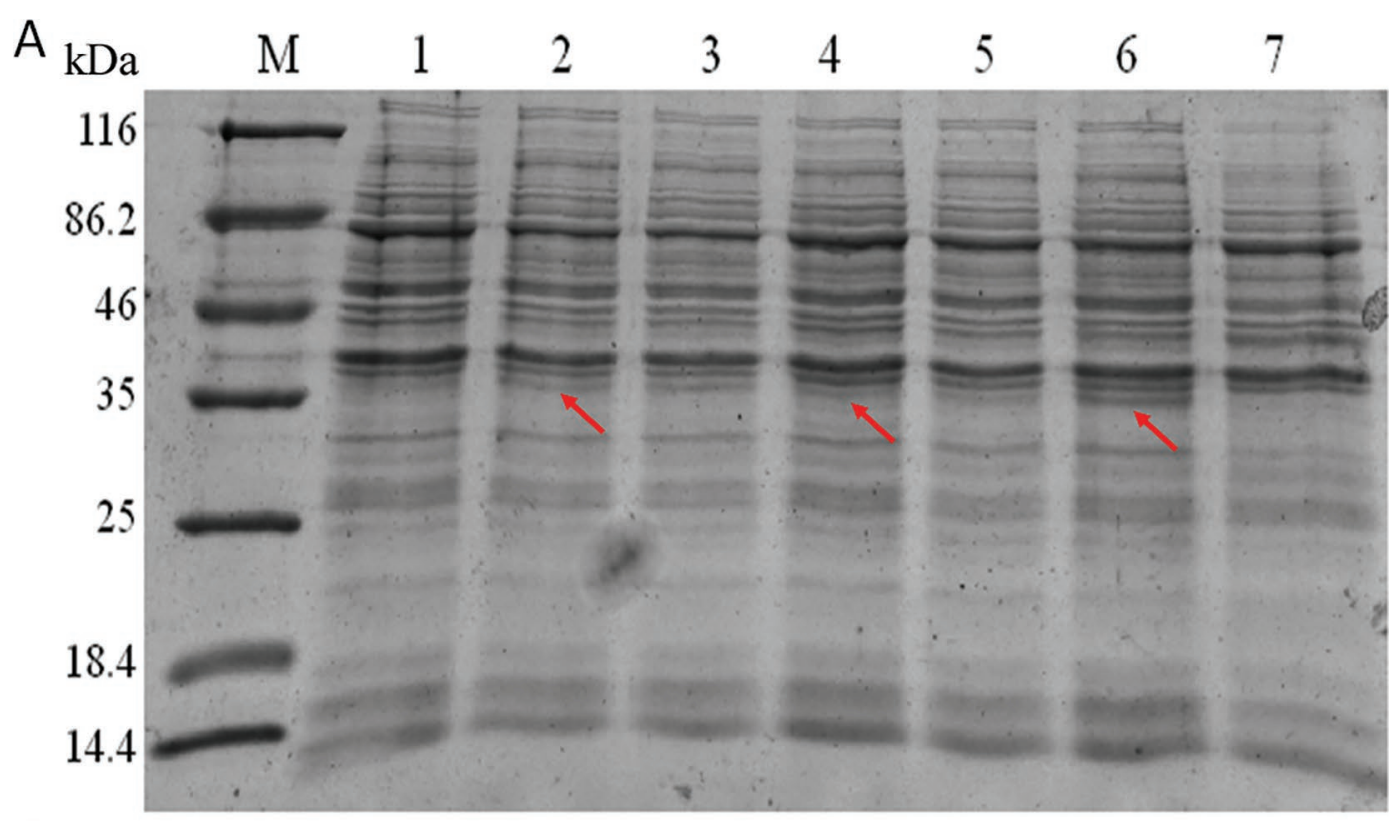

B

LC2W/pWQH01 (overexpression of BSH1)

LC2W/pWQH03 (overexpression of BSH3)

- LC2W/pWQH06 (overexpression of BSH1 and BSH3)

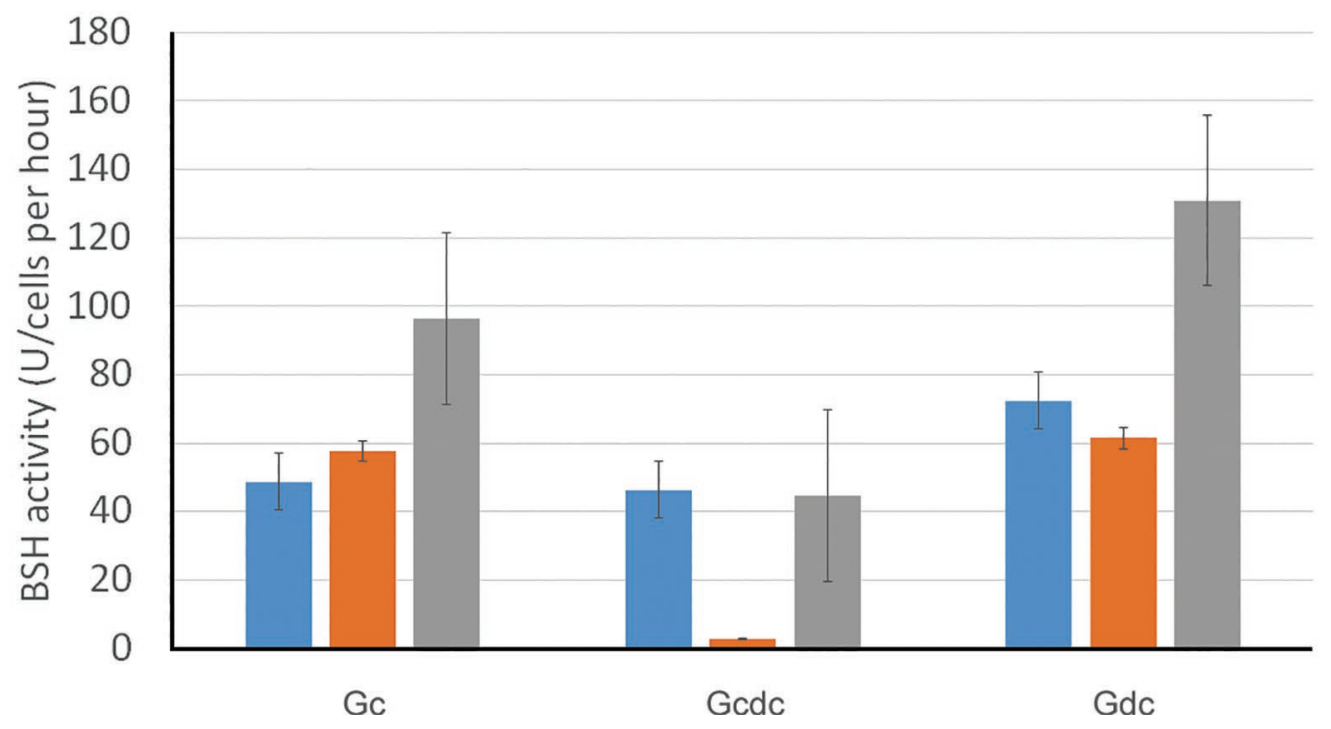

Figure 3. Overexpression of bile salt hydrolase (BSH) in Lactobacillus casei LC2W: (A) SDS-PAGE of BSH from Lactobacillus plantarum

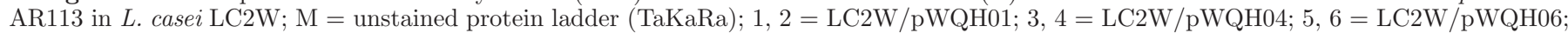
$7=$ control LC2W/pMG36e; (B) BSH activity of recombinant L. casei LC2W. Arrows indicate BSH proteins. One unit of BSH activity (U/cells per hour) was defined as the cell lysate of $10^{9}$ cells that degraded $1 \mathrm{nmol}$ of bile salt per hour. The concentrations of bile salts (glycocholate, Gc; glycodeoxycholate, Gdc; and glycochenodeoxycholate, Gcdc) in the reaction samples were detected using HPLC. Error bars indicate the SD of 3 independent measurements. Color version available online.

Indeed, BSH have been found in Bifidobacterium, Lactobacillus, Enterococcus, Bacteroides, and many other bacteria ( $\mathrm{Gu}$ et al., 2014), but not in L. casei. Our results indicated that heterologous expression of $\mathrm{BSH}$ in lactic acid bacteria could significantly enhance host activity/resistance to bile salts during industrial processes and gastrointestinal transit. In silico modeling and molecular docking could be an efficient strategy 
for rapid screening of BSH with high activity and could also be applied to the screening for other valuable enzymes in the food industry.

\section{ACKNOWLEDGMENTS}

This work was supported by the National Natural Science Foundation of China (grant No. 31371809), the New Century Excellent Talents Project of Ministry of Education of China (No. NCET-13-0901), the International Cooperation Project of Shanghai Science and Technology Commission (No. 14390711700), the "Shu Guang" project of Shanghai Municipal Education Commission and Shanghai Education Development Foundation (15SG42), and the Key Laboratory of Synthetic Biology, Chinese Academy of Sciences, China.

\section{REFERENCES}

Ai, L., Q. Guo, H. Ding, B. Guo, W. Chen, and S. W. Cui. 2016. Structure characterization of exopolysaccharides from Lactobacillus case $i \mathrm{LC} 2 \mathrm{~W}$ from skim milk. Food Hydrocoll. 56:134-143.

Ai, L., H. Zhang, B. Guo, W. Chen, Z. Wu, and Y. Wu. 2008. Preparation, partial characterization and bioactivity of exopolysaccharides from Lactobacillus casei LC2W. Carbohydr. Polym. 74:353-357.

Arnold, K., L. Bordoli, J. Kopp, and T. Schwede. 2006. The SWISSMODEL workspace: A web-based environment for protein structure homology modelling. Bioinformatics 22:195-201.

Aukrust, T. W., M. B. Brurberg, and I. F. Nes. 1995. Transformation of Lactobacillus by electroporation. Methods Mol. Biol. 47:201-208.

Bikadi, Z., and E. Hazai. 2009. Application of the PM6 semi-empirical method to modeling proteins enhances docking accuracy of AutoDock. J. Cheminform. 1:15.
Biswas, I., J. K. Jha, and N. Fromm. 2008. Shuttle expression plasmids for genetic studies in Streptococcus mutans. Microbiology 154:2275-2282.

Bordoli, L., F. Kiefer, K. Arnold, P. Benkert, J. Battey, and T. Schwede. 2009. Protein structure homology modeling using SWISS-MODEL workspace. Nat. Protoc. 4:1-13.

Chen, C., L. Ai, F. Zhou, L. Wang, H. Zhang, W. Chen, and B. Guo. 2011. Complete genome sequence of the probiotic bacterium Lactobacillus casei LC2W. J. Bacteriol. 193:3419-3420.

Dashkevicz, M. P., and S. D. Feighner. 1989. Development of a differential medium for bile salt hydrolase-active Lactobacillus spp. Appl. Environ. Microbiol. 55:11-16.

Gu, X. C., X. G. Luo, C. X. Wang, D. Y. Ma, Y. Wang, Y. Y. He, W Li, H. Zhou, and T. C. Zhang. 2014. Cloning and analysis of bile salt hydrolase genes from Lactobacillus plantarum CGMCC No. 8198. Biotechnol. Lett. 36:975-983.

Jayashree, S., S. Pooja, M. Pushpanathan, J. Rajendhran, and P. Gunasekaran. 2014. Identification and characterization of bile salt hydrolase genes from the genome of Lactobacillus fermentum MTCC 8711. Appl. Biochem. Biotechnol. 174:855-866.

Jeun, J., S. Kim, S. Y. Cho, H. J. Jun, H. J. Park, J. G. Seo, M. J. Chung, and S. J. Lee. 2010. Hypocholesterolemic effects of Lactobacillus plantarum KCTC3928 by increased bile acid excretion in C57BL/6 mice. Nutrition 26:321-330.

Kim, G. B., and B. H. Lee. 2005. Biochemical and molecular insights into bile salt hydrolase in the gastrointestinal microflora-A review. Asian-australas. J. Anim. Sci. 18:1505-1512.

Patel, A. K., R. R. Singhania, A. Pandey, and S. B. Chincholkar. 2010. Probiotic bile salt hydrolase: Current developments and perspectives. Appl. Biochem. Biotechnol. 162:166-180.

Tamura, K., G. Stecher, D. Peterson, A. Filipski, and S. Kumar. 2013. MEGA6: Molecular Evolutionary Genetics Analysis version 6.0. Mol. Biol. Evol. 30:2725-2729.

Xu, N., J. Liu, L. Ai, and L. Liu. 2015. Reconstruction and analysis of the genome-scale metabolic model of Lactobacillus casei LC2W. Gene 554:140-147. 